\title{
GCU
}

Glasgow Caledonian

University

University for the Common Good

\section{SURE based truncated tensor nuclear norm regularization for low rank tensor completion}

Morison, Gordon

Published in:

Proceedings of the 28th European Signal Processing Conference

DOI:

10.23919/Eusipco47968.2020.9287726

Publication date:

2020

Document Version

Author accepted manuscript

Link to publication in ResearchOnline

Citation for published version (Harvard):

Morison, G 2020, SURE based truncated tensor nuclear norm regularization for low rank tensor completion. in Proceedings of the 28th European Signal Processing Conference., 9287726, IEEE, pp. 2001-2005, 28th European Signal Processing Conference, Amsterdam, Netherlands, 18/01/21.

https://doi.org/10.23919/Eusipco47968.2020.9287726

\section{General rights}

Copyright and moral rights for the publications made accessible in the public portal are retained by the authors and/or other copyright owners and it is a condition of accessing publications that users recognise and abide by the legal requirements associated with these rights.

Take down policy

If you believe that this document breaches copyright please view our takedown policy at https://edshare.gcu.ac.uk/id/eprint/5179 for details of how to contact us. 


\section{GCU}

Glasgow Caledonian

University

University for the Common Good

\section{SURE Based Truncated Tensor Nuclear Norm Regularization for Low Rank Tensor Completion}

Morison, Gordon

Publication date:

2020

Document Version

Peer reviewed version

Link to publication in ResearchOnline

Citation for published version (Harvard):

Morison, G 2020, 'SURE Based Truncated Tensor Nuclear Norm Regularization for Low Rank Tensor Completion'.

\section{General rights}

Copyright and moral rights for the publications made accessible in the public portal are retained by the authors and/or other copyright owners and it is a condition of accessing publications that users recognise and abide by the legal requirements associated with these rights.

Take down policy

If you believe that this document breaches copyright please view our takedown policy at https://edshare.gcu.ac.uk/id/eprint/5179 for details

of how to contact us. 


\title{
SURE Based Truncated Tensor Nuclear Norm Regularization for Low Rank Tensor Completion
}

\author{
Gordon Morison \\ Department of Computing \\ Glasgow Caledonian University \\ Glasgow, Scotland, UK \\ gordon.morison@gcu.ac.uk
}

\begin{abstract}
Low rank tensor completion aims to recover the underlying low rank tensor obtained from its partial observations, this has a wide range of applications in Signal Processing and Machine Learning. A number of recent low rank tensor methods have successfully utilised the tensor singular value decomposition method with tensor nuclear norm minimisation via tensor singular value thresholding. This approach while proving to be effective has the potential issue that it may over or under shrink the singular values which will effect the overall performance. A truncated nuclear norm based method has been introduced which explicitly exploits the low rank assumption within the optimization in combination with tensor singular value thresholding. In this work the truncated nuclear norm approach is extended to incorporate a data driven approach based on Stein's unbiased risk estimation method which efficiently thresholds the singular values. Experimental results in a colour image denoising problem demonstrate the efficiency and accuracy of the method.
\end{abstract}

Index Terms - Truncated Tensor Nuclear Norm, Singular Value Shrinkage, SURE

\section{INTRODUCTION}

In recent years data models have played a central role in Machine Learning, Computer Vision and Signal Processing [1]. To incorporate fully the multidimensional nature of these signals has meant that a natural way of modelling this type of data is utilising tensors (multidimensional arrays). Thus the estimation of data modelled as tensors from their incomplete or corrupted observations has become increasingly important due to the enormous amount of data generated by systems such as low cost high resolution sensors, bio-informatics, imaging/video, mobile devices, sensor and social networks [2]. The key approach so far to tackle this problem has been based on exploring the inherent redundancy of the underlying modelling tensor, which has been formulated for the completion problem as a low rank estimation problem.

Beginning first with the matrix case [3], this low rank estimation can be formulated for a low rank matrix $\mathbf{Y} \in \mathbb{R}^{m \times n}$ as follows:

$$
\begin{array}{r}
\min _{\mathbf{X}} \operatorname{rank}(\mathbf{X}) \\
\text { s.t. } \mathcal{P}_{\Omega}(\mathbf{X})=\mathcal{P}_{\Omega}(\mathbf{Y})
\end{array}
$$

where $\mathbf{X} \in \mathbb{R}^{m \times n}$ and $\mathcal{P}_{\Omega}$ is an orthogonal projection operator onto the observed set $\Omega$ such that:

$$
\left[\mathcal{P}_{\Omega}(\mathbf{B})\right]_{i j}= \begin{cases}\mathbf{B}_{i j} & (i, j) \in \Omega \\ 0 & (i, j) \in \Omega^{c}\end{cases}
$$

Yet this formulation is unfortunately generally NP-hard due to the non-convex and potentially discontinuous nature of the rank function. Thus a wide range of empirical studies have demonstrated successful that a tightly convex relaxation of the rank minimisation problem is the Nuclear Norm [3]. Thus the optimization problem is reformulated as:

$$
\begin{gathered}
\min _{\mathbf{X}}\|\mathbf{X}\|_{*} \\
\text { s.t. } \mathcal{P}_{\Omega}(\mathbf{X})=\mathcal{P}_{\Omega}(\mathbf{Y})
\end{gathered}
$$

where the nuclear norm $\|\mathbf{X}\|_{*}=\sum_{k} \sigma_{k}$ is defined as the the sum of the singular values $\sigma_{k}$. The aim of this method generally is given a matrix $\mathbf{Y} \in \mathbb{R}^{m \times n}$ find a low rank matrix $\mathbf{X} \in \mathbb{R}^{m \times n}$ which satisfies the following objective function formulated as a regularized least squares problem:

$$
\hat{\mathbf{X}}=\arg \min _{\mathbf{X}}\left\|\mathcal{P}_{\Omega}(\mathbf{X})-\mathcal{P}_{\Omega}(\mathbf{Y})\right\|_{F}^{2}+\lambda\|\mathbf{X}\|_{*}
$$

the parameter $\lambda$ represents the trade off between the loss function and the low rank regularization induced by the nuclear norm. This was solved by utilizing proximal gradient descent, which is formulated as singular value thresholding [12].

Taking into account that the largest singular values contribute less to the rank of the matrix, this idea was extended by $\mathrm{Hu}$ et al. [4] to incorporate the truncated nuclear norm, which achieves a better approximation of the rank function by only considering the smallest $r$ singular values in the optimization as follows:

$$
\begin{gathered}
\min _{\mathbf{X}}\|\mathbf{X}\|_{r} \\
\text { s.t. } \mathcal{P}_{\Omega}(\mathbf{X})=\mathcal{P}_{\Omega}(\mathbf{Y})
\end{gathered}
$$

where the truncated nuclear norm $\|\mathbf{X}\|_{r}=\sum_{k=r+1}^{\min (m, n)} \sigma_{k}$ is defined as the the sum of the $\min (m, n)-r$ minimum singular values $\sigma_{k}$. This has been found to achieve a better approximation of the underlying rank function than the nuclear norm.

The success of the nuclear norm minimization methods has recently extended to the tensor case by utilizing a tensor decomposition paradigm proposed by Kilmer et al [5], the tensor singular value decomposition (t-SVD), and a promising approximation of the tensor rank which is derived from the t-SVD, defined as the tubal nuclear norm [7], which takes the sum of the nuclear norm of all front tensor slices in the Fourier 
domain. This provides a convex relaxation of the tensor rank. The optimization is give as follows:

$$
\begin{array}{r}
\min _{\mathcal{X}} \sum_{k=1}^{n_{3}}\left\|\overline{\mathbf{X}}^{(k)}\right\|_{*} \\
\text { s.t. } \mathcal{P}_{\Omega}(\mathcal{X})=\mathcal{P}_{\Omega}\left(\mathcal{X}_{0}\right)
\end{array}
$$

where $\mathcal{X} \in \mathbb{R}^{n_{1} \times n_{2} \times n_{3}}$ is a third order tensor and $\bar{X}^{(k)}$ is the $k^{t h}$ frontal slice of $\overline{\mathcal{X}}$ where $\overline{\mathcal{X}}=\operatorname{fft}(\mathcal{X},[], 3)$, and $\mathcal{X}_{0}$ is the initial tensor with missing values. Further tensor notation will be defined in Section 2.

Within this paper the work of Xue et al. [8], [9] which extended the truncated nuclear norm method to the tensor case is further developed and improved to utilize an enhanced singular value thresholding, based on the Stein Unbiased Risk Estimator (SURE) [10]. It is demonstrated that the improved SURE based singular value thresholding improves upon the previous method by experimental evaluation.

\section{TENSORS AND TENSOR NUCLEAR NORM}

Throughout this paper, tensors are denoted using calligraphic letters e.g. $\mathcal{X}$, matrices are denoted as bold face capital letters e.g. $\mathbf{X}$, vectors are denoted as bold lower case letters e.g. $\mathbf{x}$ and scalars are denoted as lower case letters e.g. $x$. Specifically a $d$-order tensor of size $n_{1} \times \cdots \times n_{d}$ is denoted as $\mathcal{X} \in \mathbb{R}^{n_{1} \times \cdots \times n_{d}}$. The Frobenius norm for a third order tensor is defined as $\|\mathcal{A}\|_{F}=\sqrt{\sum_{i j k} a_{i j k}^{2}}$ [5]. The transpose of a tensor $\mathcal{X} \in \mathbb{R}^{n_{1} \times n_{2} \times n_{3}}$ is $\mathcal{X}^{T} \in \mathbb{R}^{n_{2} \times n_{1} \times n_{3}}$ obtained by transposing the frontal slices and reversing the order of the transpose frontal slices from 2 to $n_{3}$ [6].

\section{A. Tensor Product}

To introduce the concept of a tensor product it is first useful to introduce the concept of transforming a third order tensor $\mathcal{A} \in \mathbb{R}^{n_{1} \times n_{2} \times n_{3}}$ into a block circulant matrix. Thus for a tensor $\mathcal{A}$ with frontal slices $A=\mathcal{A}(:,:, k)$ using Matlab notation of size $n_{1} \times n_{2}$ the following mapping is introduced:

$$
\operatorname{bcirc}(\mathcal{A})=\left(\begin{array}{cccc}
A^{(1)} & A^{\left(n_{3}\right)} & \cdots & A^{(2)} \\
A^{(2)} & A^{(1)} & \cdots & A^{(3)} \\
\vdots & \vdots & \ddots & \vdots \\
A^{\left(n_{3}\right)} & A^{\left(n_{3}-1\right)} & \cdots & A^{(1)}
\end{array}\right)
$$

which is a block circulant matrix of size $n_{1} n_{3} \times n_{2} n_{3}$. Introducing a function unfold which takes the frontal slices of a tensor and creates a matrix of size $n_{1} n_{3} \times n_{2}$ as follows:

$$
\operatorname{unfold}(\mathcal{A})=\left(\begin{array}{c}
A^{(1)} \\
A^{(2)} \\
\vdots \\
A^{\left(n_{3}\right)}
\end{array}\right)
$$

and the function fold undoes this operation which recreates the tensor $\mathcal{A}$ as follows:

$$
\mathcal{A}=\operatorname{fold}(\operatorname{unfold}(\mathcal{A}))
$$

Thus, following the work introduced by Kilmer et al. [5], [14] the for a tensor $\mathcal{A} \in \mathbb{R}^{n_{1} \times n_{2} \times n_{3}}$ and $\mathcal{B} \in \mathbb{R}^{n_{2} \times n_{4} \times n_{3}}$ then the tensor product $\mathcal{A} * \mathcal{B} \in \mathbb{R}^{n_{1} \times n_{4} \times n_{3}}$ is given as:

$$
\mathcal{A} * \mathcal{B} \triangleq \operatorname{fold}(\operatorname{bcirc}(\mathcal{A}) \cdot \operatorname{unfold}(\mathcal{B}))
$$

Thus exploiting the above tensor product led to the development of the tensor SVD (t-SVD) defined in the following section.

\section{B. $t-S V D$}

Utilizing the developments for the tensor product detailed above Kilmer et al. [5] proposed the Tensor Singular Value Decomposition (t-SVD) for a tensor $\mathcal{A} \in \mathbb{R}^{n_{1} \times n_{2} \times n_{3}}$ as follows:

$$
\mathcal{A} \triangleq \mathcal{U} * \mathcal{S} * \mathcal{V}^{T}
$$

where $\mathcal{U} \in \mathbb{R}^{n_{1} \times n_{1} \times n_{3}}$ and $\mathcal{V} \in \mathbb{R}^{n_{2} \times n_{2} \times n_{3}}$ and $\mathcal{S} \in$ $\mathbb{R}^{n_{1} \times n_{2} \times n_{3}}$, where each frontal slice of $\mathcal{S}$ is diagonal. Taking into account the property that block circulant matrices can be transformed to block diagonal matrices using normalized Fourier transform matrices then we have the following block diagonal representation:

$\operatorname{bdiag}\left(\overline{\mathbf{A}}^{(1)}, \ldots, \overline{\mathbf{A}}^{\left(n_{3}\right)}\right)=\left(\mathbf{F}_{n_{3}} \otimes \mathbf{I}_{n_{1}}\right) \cdot \operatorname{bcirc}(\mathcal{A}) \cdot\left(\mathbf{F}_{n_{3}}^{T} \otimes \mathbf{I}_{n_{2}}\right)$

where the function bdiag creates a block diagonal matrix, $\mathbf{F}_{n_{3}}$ denotes the $n_{3} \times n_{3}$ DFT matrix and $\otimes$ is the Kronecker product. Yet it was shown that it is not necessary to create the block diagonal matrix, the SVD can be performed on the frontal slices of the tensor in the Fourier domain. This is detailed in Algorithm 1. This development led to the

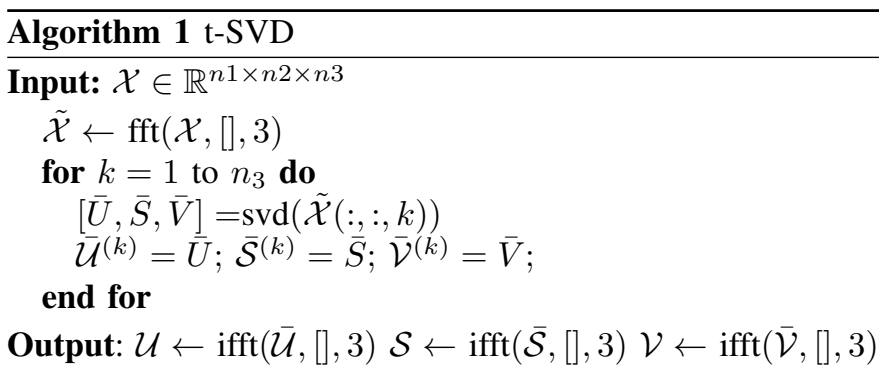

development of a new type of rank definition by taking the t-SVD of a tensor $\mathcal{A} \in \mathbb{R}^{n_{1} \times n_{2} \times n_{3}}$ as $\mathcal{U} * \mathcal{S} * \mathcal{V}^{T}$, the tensor tubal rank is defined as the maximum rank among all diagonal slices of $\mathcal{S}, \max _{k}\left(\operatorname{rank}\left(\mathbf{S}^{(k)}\right)\right)$, where $\mathbf{S}^{(k)}$ is the frontal slices of the tensor $\mathcal{S}$. Using the t-SVD leads to a definition of the tensor nuclear norm as the sum of the trace of the singular values in the frontal slices of $\mathcal{S}$ defines as follows:

$$
\|\mathcal{A}\|_{*} \triangleq \operatorname{tr}(\mathcal{S})=\sum_{k=1}^{n_{3}} \operatorname{tr}\left(\mathbf{S}^{(k)}\right)
$$

Xue et al [8], [9] exploited and proved the symmetry properties of the trace of the tensor product to demonstrate as follows:

$$
\operatorname{tr}(\mathcal{A} * \mathcal{B})=\operatorname{tr}\left(\overline{\mathbf{A}}^{(1)} \overline{\mathbf{B}}^{(1)}\right)
$$


This was then subsequently utilised to developed a simplified version of the tensor nuclear norm:

$$
\|\mathcal{A}\|_{*} \triangleq \operatorname{tr}(\mathcal{S})=\operatorname{tr}\left(\overline{\mathbf{S}}^{(1)}\right)=\left\|\overline{\mathbf{A}}^{(1)}\right\|_{*}
$$

This approach represents a significant reduction in computation in comparison to the $\mathrm{t}-\mathrm{SVD}$ based method requiring only a FFT and a SVD. This is implemented within the truncated tensor nuclear norm method detailed in the following section.

\section{TEnsor TRUnCATED Nuclear NORM}

Extending the $\mathrm{Hu}$ et al. [4] truncated nuclear norm from the matrix case to the tensor case Xue et al. [8], [9] adapted the approach within the t-SVD case by aiming to minimize the smallest $\min (m, n)-r$ singular values. Thus incorporating the above reduced complexity Fourier based tensor nuclear norm Xue et al. defined the tensor truncated nuclear norm as follows:

$$
\begin{aligned}
\|\mathcal{X}\|_{r} & \triangleq\|\overline{\mathbf{X}}\|_{r}=\sum_{k=r+1}^{\min \left(n_{1}, n_{2}\right)} \sigma_{k}\left(\overline{\mathbf{X}}^{(1)}\right) \\
& =\sum_{k=1}^{\min \left(n_{1}, n_{2}\right)} \sigma_{k}\left(\overline{\mathbf{X}}^{(1)}\right)-\sum_{k=1}^{r} \sigma_{k}\left(\overline{\mathbf{X}}^{(1)}\right)
\end{aligned}
$$

where $\overline{\mathbf{X}}^{(k)}$ represents the Fourier transform of the $k^{t h}$ frontal slices of the tensor $\mathcal{X}$. Extending the Von Neumann trace inequality based analysis defined in $\mathrm{Hu}$ et al. [4] the following truncated tensor nuclear norm function is developed:

$$
\begin{aligned}
\|\mathcal{X}\|_{r} & \triangleq\left\|\overline{\mathbf{X}}^{(1)}\right\|_{*}-\max _{\substack{\overline{\mathbf{A}}^{(1)} \overline{\mathbf{A}}^{(1) T}=\mathbf{I} \\
\overline{\mathbf{B}}^{(1)} \overline{\mathbf{B}}^{(1) T}=\mathbf{I}}} \operatorname{tr}\left(\overline{\mathbf{A}}^{(1)} \overline{\mathbf{X}}^{(1)} \overline{\mathbf{B}}^{(1) T}\right) \\
& =\|\mathcal{X}\|_{*}-\max _{\substack{\mathcal{A} * \mathcal{A}^{T}=\mathcal{I} \\
\mathcal{B} * \mathcal{B}^{T}=\mathcal{I}}} \operatorname{tr}\left(\mathcal{A} * \mathcal{X} * \mathcal{B}^{T}\right)
\end{aligned}
$$

where $\mathcal{A}$ and $\mathcal{B}$ are derived from the t-SVD. The final optimization problem proposed in the Xue et al. [8], [9] paper extending from the matrix case in $\mathrm{Hu}$ et al. [4] was given as:

$$
\begin{array}{r}
\min _{\mathcal{X}}\|\mathcal{X}\|_{*}-\operatorname{tr}\left(\mathcal{A}_{l} * \mathcal{X} * \mathcal{B}_{l}^{T}\right) \\
\text { s.t. } \mathcal{P}_{\Omega}(\mathcal{X})=\mathcal{P}_{\Omega}(\mathcal{M})
\end{array}
$$

with the full optimization procedure detailed in Algorithm 2. The tensor based version of the Alternating Direction Method of Multipliers Optimization (ADMM) based optimization method extended from $\mathrm{Hu}$ et al. [4] is detailed in Algorithm 3. Within Algorithm 3 Step 1 is solved using the singular value soft thresholding algorithm [12] which is defined as follows for a threshold $\lambda \geq 0$ :

$$
\operatorname{SVT}_{\lambda}(\mathbf{X})=\operatorname{diag}\left(\left(\sigma_{k}-\lambda\right)_{+}\right)
$$

where the operator $(t)_{+}=\max (0, t)$. This operator shrinks the singular values of towards zero. This is a mathematically appealing approach as the singular value thresholding approach represents the proximal gradient of the nuclear norm [12]. Yet this approach still requires the value of $\lambda$ to be chosen in advance. The key focus of this paper is to suggest an improved method for estimating $\lambda$ for application within the truncated tensor nuclear norm framework for tensor completion. This is detailed within the following section. $\overline{\text { Algorithm } 2 \text { Low Rank Tensor Completion via Truncated }}$ Tensor Nuclear Norm

Input: $\mathcal{M}_{l}$ incomplete data, $\Omega$ index of known elements, $\Omega^{c}$ set of unknown elements

Initialize: $\mathcal{X}_{1}=\mathcal{M}_{\Omega}, \varepsilon=10^{-3}, l=1, L=50$.

\section{1: repeat}

2: $\quad$ Step 1: $\left[\mathcal{U}_{l}, \mathcal{S}_{l}, \mathcal{V}_{l}\right]=\mathrm{t}-\operatorname{SVD}\left(\mathcal{X}_{l}\right)$

3: $\quad$ Compute $\mathcal{A}_{l}$ and $\mathcal{B}_{l}$ for $r \leq \min \left(n_{1}, n_{2}\right)$ as:

$$
\mathcal{A}_{l}=\mathcal{U}(:, 1: r,:)^{T}, \mathcal{B}_{l}=\mathcal{V}(:, 1: r,:)^{T}
$$

4: Step 2: Optimize:

$$
\begin{aligned}
& \mathcal{X}_{l+1}=\arg \min \mathbf{X}\|\mathcal{X}\|_{*}-\operatorname{tr}\left(\mathcal{A}_{l} * \mathcal{X} * \mathcal{B}_{l}^{T}\right) \\
& \text { s.t. } \mathcal{P}_{\Omega}\left(\mathcal{X}_{\Omega}\right)=\mathcal{P}_{\Omega}\left(\mathcal{M}_{\Omega}\right) \\
& l=l+1
\end{aligned}
$$

5: until $\left\|\mathcal{X}_{l+1}-\mathcal{X}_{l}\right\|_{F} \leq \varepsilon$ or $l>L$

Output: Completed tensor $\mathcal{X}_{\text {rec }}$

\section{Algorithm 3 Truncated Tensor Nuclear Norm \\ Input: $\mathcal{A}_{l}, \mathcal{B}_{l}, \mathcal{M}, \mu=5 \times 10^{-4}, \xi=10^{-4}, K=200$ \\ Initialize: $\mathcal{X}_{1}=\mathcal{M}_{\Omega}, \mathcal{W}_{1}=\mathcal{Y}_{1}=\mathcal{X}_{1}, k=1$.}

1: repeat

2: $\quad$ Step 1: $\mathcal{X}_{k+1}=\operatorname{SVT}_{\lambda}\left(\mathcal{W}_{k}-\frac{1}{\mu} \mathcal{Y}_{k}\right)$

3: $\quad$ Step 2: $\mathcal{W}_{k+1}=\mathcal{X}_{k+1}+\frac{1}{\mu}\left(\mathcal{A}_{l}^{T} * \mathcal{B}_{l}+\mathcal{Y}_{k}\right)$

4: $\quad$ Step 3: $\mathcal{Y}_{k+1}=\mathcal{Y}_{k}+\mu\left(\mathcal{X}_{k+1}-\mathcal{W}_{k+1}\right)$

Keep the values of known elements constant

$$
\begin{gathered}
\mathcal{W}_{k+1}=\left(\mathcal{W}_{k+1}\right)_{\Omega^{c}}+\mathcal{M}_{\Omega} \\
\quad k=k+1 \\
\text { 5: until }\left\|\mathcal{X}_{k+1}-\mathcal{X}_{k}\right\|_{F} \leq \xi \text { or } k>K
\end{gathered}
$$

Output: Completed tensor

\section{A. Optimization via SURE Singular Value Shrinkage}

Within the development of the Xue et al. [8], [9] algorithm the classic singular value thresholding algorithm [13] is applied with a fixed threshold value $\lambda$. This provides the question how much shrinkage should be applied to the singular values based on the data, too much will result in a large bias, whereas too litte will result in a large variance. The ideal scenario would be to minimise the mean square error or risk of the estimator, but as this would require the true low rank tensor which is unknown. Within [11] a solution to this problem was offered based on the Stein Unbiased Risk Estimator (SURE) 
[10]. This is given as follows:

$$
\begin{aligned}
\operatorname{SURE}\left(\mathrm{SVT}_{\lambda}\right)(\mathbf{X})_{\tau} & =-m n \tau^{2}+\sum_{i=1}^{\min (m, n)} \min \left(\lambda^{2}, \sigma_{i}^{2}\right) \\
& +2 \tau^{2} \operatorname{div}\left(\mathrm{SVT}_{\lambda}(\mathbf{X})\right)
\end{aligned}
$$

where as previously the term $\left\{\sigma_{k}\right\}_{k=1}^{\min (m . n)}$ are the singular values of $\mathbf{X} \in \mathbb{R}^{m \times n}, \tau^{2}$ is an estimate of the noise standard deviation, and the function div, is the divergence of the nonlinear mapping of the $\mathrm{SVT}_{\lambda}$ given as follows:

$$
\begin{aligned}
\operatorname{div}\left(\mathrm{SVT}_{\lambda}\right)(\mathbf{X}) & =\sum_{i=1}^{\min (m, n)}\left[\mathbb{I}\left(\sigma_{i}>\lambda\right)\right. \\
& \left.+|m-n|\left(1-\frac{\lambda}{\sigma_{i}}\right)_{+}\right] \\
& +2 \sum_{i, j=1 ; i \neq j}^{\min (m, n)} \frac{\sigma_{i}\left(\sigma_{i}-\lambda\right)_{+}}{\sigma_{i}^{2}-\sigma_{j}^{2}}
\end{aligned}
$$

Under mild conditions where there are no repeated singular values, which is normally valid in practise Equation 20 provides a method to determine a suitable threshold value $\lambda$ which minimises the estimate of the risk. This formulation is utilised to extend upon the Xue et al. [8], [9] truncated nuclear norm minimization method as detailed in Algorithm 4. The

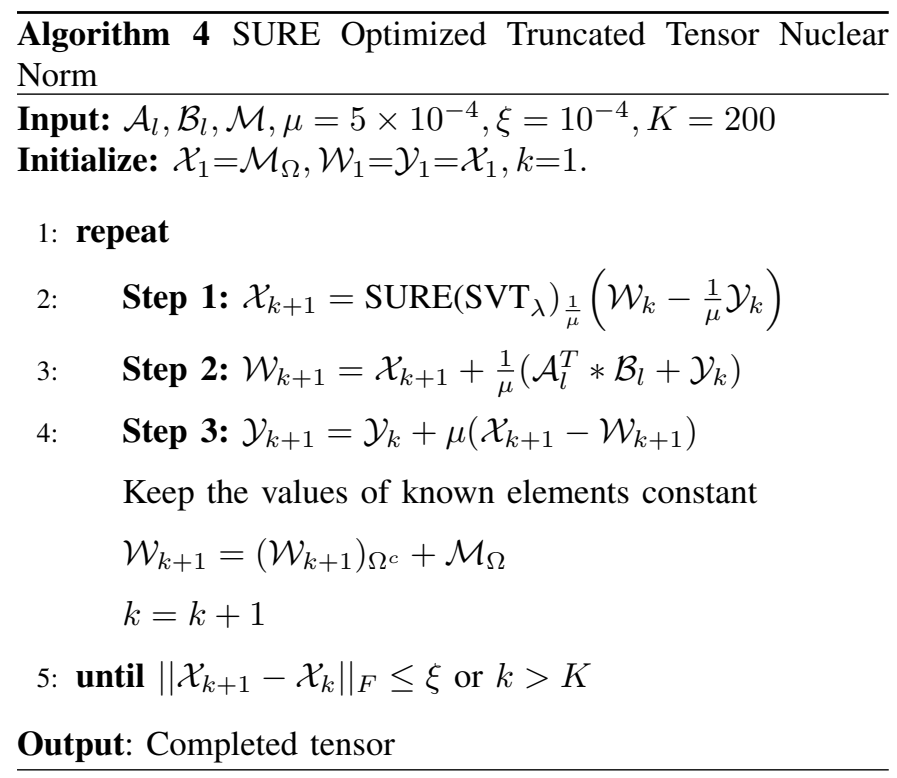

algorithm detailed within this section follows directly from the approach detailed within Xue et al [8], [9], extending Step 1 of Algorithm 3 with the SURE based optimized shrinkage function as shown in Algorithm 4. An evaluation of the approach for color image recovery is detailed in the following section.

\section{EXPERIMENTS}

To demonstrate the accuracy and efficiency of the proposed algorithm the following approach is undertaken as defined within the $\mathrm{Xu}$ et al. paper. 10 colour images shown in Figure 1 of size $400 \times 300$ are utilized. For the experiment $50 \%$ of the pixels within the image are randomly deleted. The aim in this setting of the algorithm is to recover the missing multi-dimensional pixels. The results of the algorithm
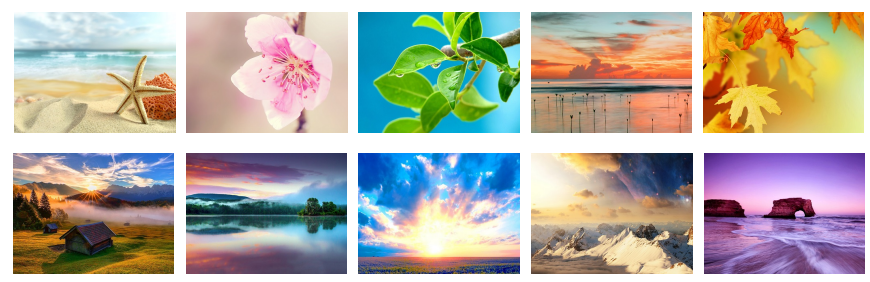

Fig. 1: Experimental Colour Image Examples

for consistency is compared with the following tensor based methods:

- Matrix Completion by Truncated Nuclear Norm Regularization (TNNR) [4]

- Tensor Completion by Sum of Matricized Nuclear Norm (SMNN) [16]

- Adaptive Tensor Nuclear Norm Minimization (ATNN) [17]

- Tensor Completion by Tubal-NN (Tubal-NN) [7]

- Tensor Completion by Truncated Nuclear Norm [9]

- Tensor Completion via SURE based Truncated Nuclear Norm (ours)

To assess the performance of the algorithms the peak signalto-noise ratio (PSNR) is utilized as the performance metric, which is defined as follows:

$$
\begin{gathered}
M S E=\frac{\left\|\left(\mathcal{X}_{\text {rec }}-\mathcal{M}\right)_{\Omega}\right\|_{F}^{2}}{T} \\
P S N R=10 \log _{10}\left(\frac{255^{2}}{M S E}\right)
\end{gathered}
$$

where $\mathrm{T}$ is the total number of elements that are missing from the image, and the maximum pixel value in the color image is 255. It should be noted that only the ADMM based method of Xue et al. is included, this is because while the Accelerated Proximal Gradient Line Search (APGL) method defined in the paper demonstrated improved convergence with respect to the ADMM method the PSNR results were less competitive. From the experimental results it is clear that the inclusion of the SURE based singular value shrinkage within the tSVD and ADMM optimized framework positively improves the PSNR and decreases the number of iterations required for convergence, at the cost of some minimal additional computation for the optimization of the SURE estimation.

\section{CONClusion}

Within this paper, an extension to the truncated tensor nuclear norm method has been presented for application to the low rank tensor completion problem. This approach deviates from previous tensor nuclear norm methods by optimizing the truncated nuclear norm using the ADMM method and a SURE 
TABLE I: PSNR and iterations in parentheses for each algorithm

\begin{tabular}{|c|c|c|c|c|c|c|}
\hline No. & TNNR & SMNN & ATNN & Tubal-NN & T-TNN & ST-TNN \\
\hline \multirow{2}{*}{1} & 25.56 & 22.19 & 25.04 & 28.91 & 29.65 & 30.79 \\
\hline & (665) & (343) & (285) & (264) & (165) & (105) \\
\hline \multirow{2}{*}{2} & 28.79 & 23.19 & 27.43 & 31.45 & 32.55 & 32.95 \\
\hline & (541) & (346) & (287) & (258) & (148) & (107) \\
\hline \multirow{2}{*}{3} & 24.33 & 22.23 & 24.42 & 26.17 & 27.67 & 27.25 \\
\hline & (878) & (341) & (291) & (262) & (178) & (155) \\
\hline \multirow{2}{*}{4} & 30.82 & 26.40 & 28.97 & 34.19 & 35.32 & 35.27 \\
\hline & (614) & (351) & (276) & (244) & (181) & (106) \\
\hline \multirow{2}{*}{5} & 28.96 & 24.95 & 26.34 & 30.29 & 31.20 & 31.43 \\
\hline & (656) & (346) & (286) & (246) & (180) & (158) \\
\hline \multirow{2}{*}{6} & 23.23 & 22.95 & 23.60 & 25.48 & 26.24 & 27.25 \\
\hline & (813) & (339) & (277) & (241) & (212) & (161) \\
\hline \multirow{2}{*}{7} & 30.55 & 27.85 & 29.05 & 33.55 & 34.45 & 34.60 \\
\hline & (624) & (345) & (283) & (247) & (188) & (164) \\
\hline \multirow{2}{*}{8} & 26.04 & 22.80 & 25.68 & 29.02 & 29.93 & 29.93 \\
\hline & (573) & (344) & (278) & (249) & (173) & (152) \\
\hline \multirow{2}{*}{9} & 24.17 & 22.53 & 24.12 & 27.92 & 28.98 & 29.86 \\
\hline & (639) & (347) & (283) & (260) & (140) & (159) \\
\hline \multirow{2}{*}{10} & 26.60 & 22.40 & 26.28 & 31.59 & 32.60 & 33.57 \\
\hline & (745) & (349) & (284) & (254) & (184) & (109) \\
\hline
\end{tabular}

based singular value thresholding method. The experimental results demonstrate that the method improves upon the previously developed truncated tensor nuclear norm method for the case of colour image recovery. A limitation of the method is it is constrained to third order tensors. Future developments will include further optimization of the singular value shrinkage functions and extensions of the approach to $\mathrm{N}$-way tensors $(N>3)$.

\section{REFERENCES}

[1] N. D. Sidiropoulos, L. De Lathauwer, X. Fu, K. Huang, E. E. Papalexakis and C. Faloutsos, "Tensor Decomposition for Signal Processing and Machine Learning," IEEE Transactions on Signal Processing, vol. 65, no. 13 , pp. $3551-3582,2017$

[2] A. Cichocki et al., "Tensor decompositions for signal processing applications: From two-way to multiway component analysis," IEEE Signal Processing Magazine, vol. 32, no. 2, pp. 145-163, 2015

[3] E. J. Candes and B. Recht, "Exact matrix completion via convex optimization", Foundations of Computational Mathematics, vol. 9, no. 6, pp. 717-772, 2009

[4] Y. Hu, D. Zhang, J. Ye, X. Li and X. He, "Fast and Accurate Matrix Completion via Truncated Nuclear Norm Regularization," IEEE Transactions on Pattern Analysis and Machine Intelligence, vol. 35, no. 9, pp. 2117-2130, 2013

[5] M. E. Kilmer, C. D. Martin, L. Perrone, "A third-order generalization of the matrix SVD as a product of third-order tensors", Tech. Report Technical Report TR-2008-4, Tufts University, Department of Computer Science, 2008

[6] M. E. Kilmer, K. Braman, N. Hao, and R. C. Hoover, "Third-order tensors as operators on matrices: A theoretical and computational framework with applications in imaging," SIAM J. Matrix Analysis and Applications, vol. 34, no. 1, pp. 148-172, 2013.

[7] Z. Zhang, G. Ely, S. Aeron, N. Hao, M. Kilmer, "Novel methods for multilinear data completion and de-noising based on tensor-SVD", International Conference on Computer Vision and Pattern Recognition, IEEE pp. 3842-384 2014

[8] S. Xue, W. Qiu, F. Liu, X. Jin, ”Low-rank tensor completion by truncated nuclear norm regularization", 24th International Conference on Pattern Recognition, IEEE, Beijing, China, pp. 1-6. 2018

[9] S. Xue, W. Qiu, F. Liu, X. Jin. "Truncated nuclear norm regularization for low-rank tensor completion arXiv preprint arXiv:1901.01997, 2019.
[10] C. M. Stein. "Estimation of the mean of a multivariate normal distribution", The Annals of Statistics, 9(6):pp. 1135-1151, 1981

[11] E. J. Candes, C. A. Sing-Long, J. D. Trzasko, "Unbiased Risk Estimates for Singular Value Thresholding and Spectral Estimators," in IEEE Transactions on Signal Processing, vol. 61, no. 19, pp. 4643-4657, 2013.

[12] J.-F. Cai, E. J. Candes, and Z. Shen. "A singular value thresholding algorithm for matrix completion", SIAM Journal on Optimization, 2010

[13] E. T. Hale, W. Yin, and Y. Zhang. "Fixed-point continuation for 11minimization: Methodology and convergence", SIAM Journal on Optimization, 2008.

[14] M. E. Kilmer and C. D. Martin, "Factorization strategies for third-order tensors", Linear Algebra and its Applications, Special Issue in Honor of G. W. Stewart's 70th birthday 2010

[15] K. Braman, "Third-order tensors as linear operators on a space of matrices", Linear Algebra and its Applications, pp. 1241-1253 2010

[16] J. Liu, P. Musialski, P. Wonka, J. Ye, "Tensor completion for estimating missing values in visual data", IEEE Transactions on Pattern Analysis and Machine Intelligence. 35 (1) 208-220. 2018

[17] C. Zhang, W. Hu, T. Jin, Z. Mei, "Nonlocal image denoising via adaptive tensor nuclear norm minimization", Neural Computing and Applications 29 (1) 3-19. 2018 